\title{
Nature and strength of bonding in a crystal of semiconducting nanotubes: van der Waals density functional calculations and analytical results
}

\author{
Kleis, Jesper; Schröder, Elsebeth; Hyldgaard, Per
}

Published in:

Physical Review B Condensed Matter

Link to article, DOI:

10.1103/PhysRevB.77.205422

Publication date:

2008

Document Version

Publisher's PDF, also known as Version of record

Link back to DTU Orbit

Citation (APA):

Kleis, J., Schröder, E., \& Hyldgaard, P. (2008). Nature and strength of bonding in a crystal of semiconducting nanotubes: van der Waals density functional calculations and analytical results. Physical Review $B$ Condensed Matter, 77(20), 205422. https://doi.org/10.1103/PhysRevB.77.205422

\section{General rights}

Copyright and moral rights for the publications made accessible in the public portal are retained by the authors and/or other copyright owners and it is a condition of accessing publications that users recognise and abide by the legal requirements associated with these rights.

- Users may download and print one copy of any publication from the public portal for the purpose of private study or research.

- You may not further distribute the material or use it for any profit-making activity or commercial gain

- You may freely distribute the URL identifying the publication in the public portal 


\title{
Nature and strength of bonding in a crystal of semiconducting nanotubes: van der Waals density functional calculations and analytical results
}

\author{
Jesper Kleis, ${ }^{1,2}$ Elsebeth Schröder, ${ }^{1}$ and Per Hyldgaard ${ }^{3, *}$ \\ ${ }^{1}$ Department of Applied Physics, Chalmers University of Technology, SE-412 96 Göteborg, Sweden \\ ${ }^{2}$ CAMD, Department of Physics, Technical University of Denmark, DK-2800 Kgs. Lyngby, Denmark \\ ${ }^{3}$ Department of Microtechnology and Nanoscience, MC2, Chalmers University of Technology, SE-412 96 Göteborg, Sweden
}

(Received 16 January 2008; revised manuscript received 24 March 2008; published 15 May 2008)

\begin{abstract}
The dispersive interaction between nanotubes is investigated through ab initio theory calculations and in an analytical approximation. A van der Waals density functional (vdW-DF) [M. Dion et al., Phys. Rev. Lett. 92, 246401 (2004)] is used to determine and compare the binding of a pair of nanotubes as well as in a nanotube crystal. To analyze the interaction and determine the importance of morphology, we further compare results of our $a b$ initio calculations to a simple analytical result that we obtain for a pair of well-separated nanotubes. In contrast to traditional density functional theory calculations, the vdW-DF study predicts an intertube vdW bonding with a strength that is consistent with recent observations for the interlayer binding in graphitics. It also produces a nanotube wall-to-wall separation, which is in very good agreement with experiments. Moreover, we find that the vdW-DF result for the nanotube-crystal binding energy can be approximated by a sum of nanotube-pair interactions when these are calculated in vdW-DF. This observation suggests a framework for an efficient implementation of quantum-physical modeling of the carbon nanotube bundling in more general nanotube bundles, including nanotube yarn and rope structures.
\end{abstract}

DOI: 10.1103/PhysRevB.77.205422

PACS number(s): 61.50.Lt, 61.46.-w, 71.15.Mb

\section{INTRODUCTION}

Carbon nanotubes (CNTs) have a wealth of exciting physical properties that have made them the focus for a very broad range of fundamental-science studies. ${ }^{1}$ The CNTs have, for example, an exceptionally large Young modulus. ${ }^{2}$ The individual CNTs have nanoscale diameters and micron scale lengths but a range of CNT assembly processes promise technology applications even on more macroscopic scales. Thermal treatment can cause a fullerene source to transform into a highly regular CNT crystal with parallel tubes aligned in a hexagonal structure. ${ }^{3}$ The tubes can also form CNT bundles ${ }^{4-7}$ in which essentially parallel CNTs still have a very high degree of (local) order. The bundles can be spun into yarn ${ }^{8,9}$ and further twisted to produce torque-free ropes of micrometer diameter (and arbitrary length). The yarn and ropes have a large strength and a unique ability to absorb elastic energy in reversible extensions. ${ }^{9}$ By preselecting the nanotube source material, for example, as singlewalled CNTs, ${ }^{10}$ it is possible to ensure specific physical properties (such as metallic conductivity) also of the resulting well-aligned yarn. ${ }^{9,11}$

The science ${ }^{1-7}$ and technology progress ${ }^{8-11}$ challenges us to present a quantum-physical characterization of the bonding in the nanotube crystal ${ }^{3}$ and, by extension, in the bundles. It is valuable to have a method for parameterfree characterization of general CNT bundles and it is important to test the accuracy of available computational tools. The CNT crystals (and bundles) are (approximately) periodic and have a relatively simple order. This makes them accessible to calculations in density functional theory (DFT), which, in principle, provides quantum-physical accounts of general material bonding. It is straightforward to provide quantum-physical calculations for a parameter-free characterization of the intra-CNT electronic and atomic organization using traditional implementations of state-of-the-art
DFT calculations. ${ }^{12,13}$ These calculations use either the local density approximation ${ }^{14}$ (LDA) and/or the semilocal generalized gradient approximation (GGA), for example, as parametrized in the Perdew-Burke-Ernzerhof flavor. ${ }^{15}$ However, the CNT crystals and bundles are graphitic materials and the intertube attraction is known to be dominated by relatively soft dispersive or van der Waals (vdW) interactions. ${ }^{3,16}$ Neither LDA nor GGA provide any physicsbased account of the bonding between the CNTs. ${ }^{17-21}$

In this paper, we use a recently developed van der Waals density functional ${ }^{22,23}$ (vdW-DF) to provide a quantumphysical account of the vdW bonding in a hexagonal crystal ${ }^{3}$ of parallel semiconducting nanotubes. We perform state-ofthe-art DFT calculations of the intrananotube structure within GGA and of the internanotube binding within vdWDF. The study testifies to the strength of this vdW bonding, which is normally described as soft but nevertheless contributes significantly to the cohesion of the CNT crystal. Our results allow a test of the vdW-DF theory method by comparison against structure measurements for the highly ordered CNT crystal ${ }^{3}$ and bundle ${ }^{6}$ structures. The study supplements a recent vdW-DF calculation ${ }^{24}$ on a simple polymer, polyethylene, for which there also exists experimental characterization of the crystalline structure. ${ }^{25}$ It also supplements vdW-DF calculations of the benzene and DNA base-pair interactions $^{26}$ in a wider program on calculating dispersive interactions in carbon and organic materials. We provide a parameter-free theory determination of the CNT bonding in the crystal and between pairs of parallel nanotubes and document that a summation of nanotube-pair-interaction energies (calculated in vdW-DF) represents a fair approximation for the vdW-DF results for the crystal bundling energy. We also detail the nature of the mutual CNT interactions by identifying a set of distinct vdW interaction regimes. We show that the vdW interaction is significantly enhanced at the bonding separation compared with the value estimated from the asymptotic interaction. 
The vdW-DF calculations correct the accuracy problems arising in traditional state-of-the-art implementations of DFT (that use LDA or GGA) without loss of the traditional-DFT scaling $^{23}$ [computation cost increasing $\sim \mathcal{O}\left(N^{3}\right)$ with system size]. DFT calculations in GGA show no meaningful binding. ${ }^{27}$ While LDA calculations can mimic the CNT binding, it underestimates the binding separation. For the CNT bundles, the LDA result ${ }^{28}$ for the wall-to-wall separation, $\Delta_{\mathrm{LDA}}=3.1 \AA$, is $10 \%$ shorter than the experimental value, $\Delta_{\mathrm{CNT}}=3.4 \AA$. Moreover, the LDA result, ${ }^{28} \approx 10 \mathrm{meV} /$ atom, for the intertube binding in a crystal of metallic $(6,6)$ CNTs is significantly smaller than the estimate ${ }^{29}(50 \mathrm{meV} /$ atom for graphitics materials) extracted from measurements of the binding of polyaromatic hydrocarbon (PAH) molecules on graphite. The vdW-DF method corrects those problems without loss of scaling advantages by supplementing the LDA for correlation with a nonlocal contribution ${ }^{22}$ that scales like $\mathcal{O}\left(N^{2}\right)$ with the system size. ${ }^{23}$ The vdW-DF method clearly has a better scaling than implementations of canonical Möller-Plesset perturbation theory (MP2) for extended structures such as polymer crystals. ${ }^{30}$ Specially adapted-MP2 implementations can achieve a linear scaling with size for large molecules. ${ }^{31}$ The adapted-MP2 method has also been applied to extended one-dimensional systems. ${ }^{32}$ It is unclear how the adapted-MP2 evaluation of correlation and the $v d W-D F$ determination of nonlocal correlation compare in actual computing cost for extended structures such as polymer crystals ${ }^{24,32}$ and for large bulk and surface-adhesion systems. ${ }^{21,33-35}$ In any case, the complete MP2 calculation ${ }^{32}$ also involves Hartree-Fock calculations that scale worse than general DFT implementations (including, for example, vdW-DF).

The paper is organized as follows. In Sec. II, we identify regimes of interactions in crystals of nanotubes and discuss qualitative differences in the vdW bonding of semiconducting and of metallic nanotubes. Section III presents a summary of the vdW-DF calculation method that we use to obtain an $a b$ initio characterization of the semiconductingnanotube crystal. In Sec. IV, we present an analytical description of the nanotube interaction, and in Sec. V, we discuss both the strength and nature of the internanotube interaction. Section VI contains our conclusions and acknowledgments.

\section{REGIMES OF NANOTUBE INTERACTIONS}

There are several regimes of interactions relevant for the cohesion of the nanotube crystals. The individual tubes are held together by exceptionally strong covalent bonds between neighboring carbon atoms separated by just $1.42 \AA$. The binding between the CNTs is instead dominated by the $\mathrm{vdW}$ interaction that binds the tubes at a wall-to-wall CNT separation of $3.4 \AA$. The vdW interaction also causes an intertube attraction even at asymptotic distances. There are qualitative differences in the $\mathrm{vdW}$ forces in the asymptotic regime (where the interaction is defined by the dipolar electrodynamical response) and at bonding separation in graphitic materials (where multipolar contributions are documented to enhance the interaction). ${ }^{20}$
Moreover, the vdW interaction between extended semiconducting and metallic structures (for example, semiconducting and metallic CNTs) is qualitatively different, at least in the regime of asymptotic interactions. ${ }^{23,36-39}$ Singlewalled CNTs can exhibit both a semiconducting and a metallic nature of conduction depending on their chirality. Being low-dimensional systems, the semiconducting and metallic CNTs therefore exhibit significant differences in their electronic response and, consequently, in the asymptotic $\mathrm{vdW}$ interactions. ${ }^{36-39}$ In the strictly asymptotic regime, it is possible to view the CNT as wires. For a pair of insulating or semiconducting wires, the asymptotic form of the interaction is known to eventually acquire a $d^{-5}$ dependence with the separation $d$ between the wire centers of mass. In contrast, Dobson et al..$^{39}$ recently used a coupled-plasmon expansion and approximations valid for asymptotic mutual separations to derive a mutual interaction energy with a $-d^{-2}[\log (d)]^{-3 / 2}$ asymptotic scaling for metallic wires. It is not known to what extent qualitative differences between the vdW binding of metallic and semiconducting nanotubes persist down to distances relevant for their binding in CNT pairs or bundles, ${ }^{23}$ but that is an important question beyond the present scope. Extraction of CNT binding energies from the metallic-wire study Ref. 39 is complicated because there are two convoluted interaction effects arising as the CNTs approach each other. First, the CNT morphology (a hollow cylinder) manifests itself $\mathrm{f}^{40,41}$ even when the long-wavelength form of the mutual dielectric response remains applicable. Second, the nature of the van der Waals interaction changes ${ }^{20}$ so that it is no longer dominated by the long-wavelength response form but also retains interaction contributions defined by the multipole response. ${ }^{20}$

Figure 1 shows schematics of the electronic, (intratube) atomic, as well as (intertube) crystalline ordering (bundling) of nanotubes. We study the mutual binding of pairs and bundles of CNTs that have a chirality vector ${ }^{1}(8,0)$. These CNTs have a diameter a little larger than $0.6 \mathrm{~nm}$, a fourfold rotational symmetry, and an along-axis structure repeating itself every 32 atoms. Confirming also previous investigations, ${ }^{13}$ we find that state-of-the-art DFT calculations using GGA provide an excellent account of the intrananotube structural organization and electronic properties such as the nature of conduction. We use the traditional-DFTGGA results, obtained in a plane-wave implementation, ${ }^{42}$ as the starting point for vdW-DF calculations of the intertube binding. 22,23

Recent density functional approximations ${ }^{20,22,23,43-49}$ extend traditional DFT to provide a seamless, parameter-free characterization of the $\mathrm{vdW}$ binding without introducing double counting at separations with finite overlap of electron densities. In our vdW-DF method, ${ }^{22,23}$ we extract the exchange from GGA calculations but supplement the local density approximation for the correlation energy by a nonlocal correlation energy contribution $E_{c}^{\mathrm{nl}}$. This contribution is evaluated from the electron densities of the underlying traditional DFT calculations in GGA. This vdW-DF description remains applicable and effective even for large extended systems that are accessible for standard ab initio DFT calculation (although at an increase in computing cost). In fact, the vdW-DF method exhibits the same scaling as the underlying 


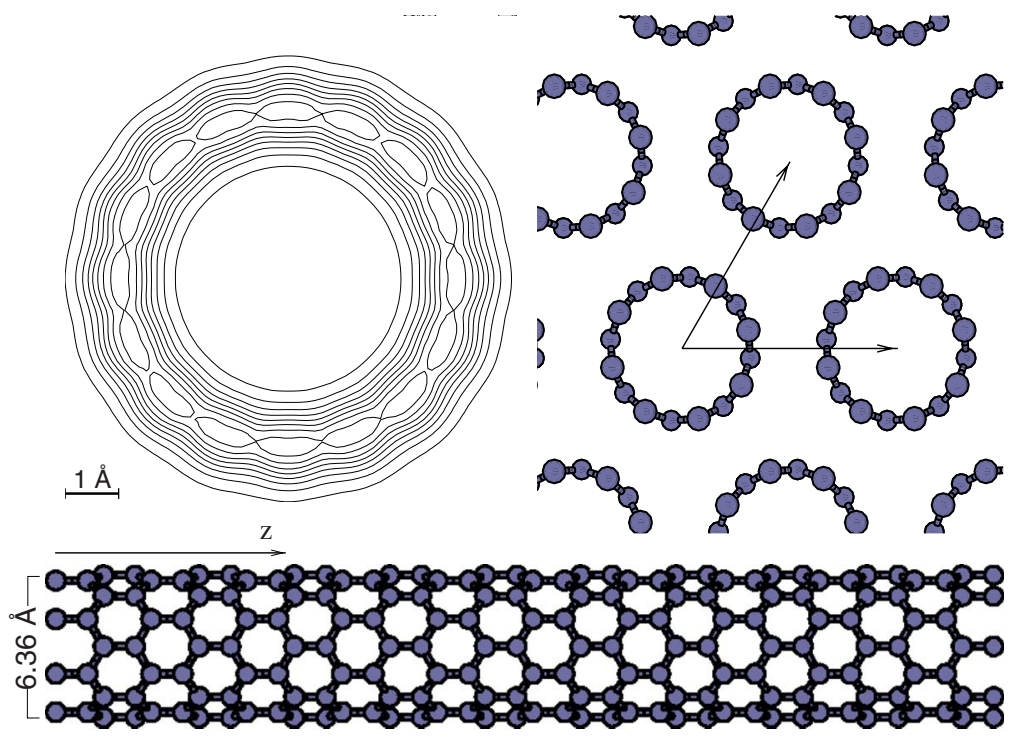

FIG. 1. (Color online) Atomic and electronic structures as well as filament organization in crystals of semiconducting $(8,0)$ zigzag nanotubes. The lower panel shows the fully relaxed atomic configuration of the individual $(8,0)$ nanotubes as calculated in a traditional implementation of $a b$ initio DFT. The top left panel shows our corresponding traditional-DFT results for the length-averaged electron density concentrated at the atomic nuclei; the contour spacing is specified in steps of $0.15 e \AA^{3}$. Finally, the top right panel shows the hexagonal crystalline order of the nanotube bundle. We calculate the intertube dispersive interaction and determine the nanotube crystalline structure by using a recently developed $a b$ initio van der Waals density functional approach (Ref. 22).

traditional-DFT calculations. ${ }^{23}$ The vdW-DF approach permits $a b$ initio characterizations of large bulk systems, for example, produced by potassium intercalation. ${ }^{21}$ It further permits $a b$ initio investigations of very large surfaceadhesion systems, for example, graphite adsorption of PAH molecules. ${ }^{33,34}$ In a controlled approximation, it even permits an $a b$ initio study (in a repeated unit cell containing 146 atoms) of the adhesion of graphite sheets on SiC surfaces. ${ }^{35}$

The vdW-DF evaluation of the nonlocal correlations $E_{c}^{\mathrm{nl}}$ (vdW interaction energy) involves a density-weighted integration of a kernel ${ }^{22}$ that contains a rich account of the complex electrodynamics. ${ }^{20,22,23,44}$ Our vdW-DF is not developed to include an explicit account of the asymptotic interaction between extended metallic one-dimensional systems. ${ }^{23}$ The form of the $\mathrm{vdW}-\mathrm{DF} \mathrm{kernel}^{22,23}$ ensures the correct asymptotic behavior of vdW interactions for atoms, molecules, and most surface and bulk systems. It also describes the asymptotic interactions for extended low-dimensional systems that are isolating or semiconducting. The form of the vdW-DF kernel ensures the correct asymptotic form of the interaction between pairs and within crystals of the $(8,0)$ CNTs because these are robustly semiconducting (characterized by a significant gap ${ }^{1}$ ). More importantly, our vdW-DF calculations of the $(8,0)$-CNT binding not only eventually reproduces a $d^{-5}$ dependence in the interaction energies but reveals a much finer structure and remains fully applicable at general separations.

To interpret this rich structure in CNT binding-energy variation with CNT separation, we also present in this paper an analytical evaluation of the CNT-pair interaction, which is valid at intermediate-to-asymptotic distances. Our analysis tool (but not our full vdW-DF calculations) makes assumptions of nonoverlapping electron densities and of a longwavelength form of the CNT dielectric response but it respects the CNT morphology. ${ }^{41,50,51}$ Comparison with the full vdW-DF calculations, therefore, allows us to deconvolute effects arising from the change in nature of the dielectric response. We thereby provide an analysis that splits the interaction into two major regimes: (1) a close regime at or near binding separations where full $a b$ initio vdW-DF calculations are essential for an accurate account and (2) an intermediateto-asymptotic regime where the long-wavelength dielectric response remains applicable but where the CNT morphology specifies the variation of the interaction. The result of this analysis documents that the vdW interaction enhances at bonding separations compared with estimates that can be extracted from knowledge of the asymptotic form of the internanotube bonding.

\section{COMPUTATIONAL METHODS}

The top right panel of Fig. 1 shows a schematic of the repeated two-dimensional hexagonal array of the nanotube bundles. We apply the vdW-DF ${ }^{22}$ method to include the dispersive interaction within the framework of traditional planewave DFT both for dimers of nanotubes, as well as for an infinitely extended nanotube crystal (Fig. 1). A selfconsistent formulation of vdW-DF has recently been derived, implemented, and tested. ${ }^{23}$ Here, we use the original, nonself-consistent (post-GGA) implementation ${ }^{22}$ that rests upon and utilizes traditional-DFT calculations to obtain the electron-density variations.

In the present vdW-DF study, we furthermore take advantage of the success of the traditional semilocal (GGA) density functionals to describe the intramolecular properties of the nanotubes (as well as the electron densities). It is, in principle, possible to provide an all-vdW-DF characterization of the intrananotube atomic structure (allowing relaxation under vdW-DF forces ${ }^{23}$ ) but the computation costs would be large. Our previous experience from an all-vdW-DF characterization of a single graphite sheet ${ }^{21}$ suggests that only minute differences would result for the CNT structure if we replaced the GGA intratube characterization by a full vdW-DF characterization.

\section{A. Nanotube atomic and electronic structure}

In the actual vdW-DF implementation, a large set of stateof-the-art traditional-DFT calculations determine the atomic structure (of the individual nanotube) and the electron- 
density variation of the nanotubes when isolated, when assembled into a hexagonal crystalline structure, or when aligned as a parallel- nanotube dimer. We use a plane-wave code $^{42}$ with ultrasoft pseudopotentials and a $1 \times 1 \times 8$ Monkhorst-Pack ${ }^{52} k$-point sampling of the Brillouin zone of the periodically repeated unit cell containing 32 atoms per nanotube. We perform self-consistent calculations in the Perdew-Burke-Ernzerhof (PBE) flavor ${ }^{15}$ of GGA for the exchange and correlation functional. We choose a plane-wave energy cutoff of $476 \mathrm{eV}$ and specify the fast Fourier transform (FFT) grid so that the (density) grid spacing remains smaller than $0.14 \AA$ in all directions.

We first determine the atomic structure of the individual $(8,0)$ nanotube, as shown in the lower panel of Fig. 1. We use our $a b$ initio calculations of the strong intrananotube atomic forces to relax the morphology to a total residual atomic force of $0.05 \mathrm{eV} / \AA$ per unit cell. The structure is characterized by an effective geometrical radius $R_{\text {geo }}$ specified as the average distance of the carbon nuclei from the axis defined by the nanotube center of mass. We determine the value of that geometric radius to be $R_{\text {geo }}=3.18 \AA$. As explained above, the atomic structure (obtained in PBE-GGA for the isolated nanotube) is kept frozen in all subsequent calculations (for nanotube-crystal and dimer cases). We have explicitly tested that no additional intratube atomic relaxation is relevant at (or beyond) the separation that characterizes the nanotube-crystal/dimer binding in our subsequent post-GGA vdW-DF characterization.

Next, we determine the electronic structure from traditional-DFT calculations for the isolated nanotubes, for the nanotube bundles, and for the dimer structures. The upper left panel of Fig. 1 shows contours (at $0.15 e \AA^{-3}$ interval) in the isolated-nanotube electron-density variation averaged along the nanotube axis. The maximum in electron density coincides with the radial position of nuclei and naturally identifies the nanotube wall. To characterize the internanotube vdW-dominated binding, we further calculate the electron density for nanotubes in hexagonal crystals and dimers as a function of the wall-to-wall nanotube separation $\Delta$ and as a function of the nanotube-rotation angle (relative rotation angle in the case of the dimer study).

We find that there are important electron-density overlaps for $\Delta<4 \AA$ when the self-consistent GGA electron densities differ from a superposition of individual-nanotube electron densities. The existence of these intertube electron-density overlaps has direct consequences for the details of the intertube vdW binding. However, the electron-density overlap does not reflect the existence of any relevant (and physically meaningful) binding arising within the GGA calculations themselves (in any of the GGA flavors).

\section{B. van der Waals density functional theory}

Like the LDA and GGA functionals, the vdW-DF is defined by approximations for the exchange and correlation energies. The nonlocal dispersive interactions responsible for keeping the bundle of nanotubes together are included as a significant extension (correction) of the correlation energy in the underlying GGA calculations. Specifically, we com- pletely replace the GGA description of correlation but use the self-consistent GGA result for the electron density to evaluate a new correlation energy that includes the nonlocal nature of the vdW binding.

Our vdW-DF splits the correlation up into local and nonlocal contributions, ${ }^{22,43}$

$$
E_{c} \approx E_{c}^{\mathrm{LDA}}+E_{c}^{\mathrm{nl}},
$$

with the local part approximated in the LDA. The nonlocal correlation energy is expressed ${ }^{22,43}$ as

$$
E_{c}^{\mathrm{nl}}=\int_{0}^{\infty} \frac{d u}{2 \pi} \operatorname{tr}[\ln (1-V \tilde{\chi})-\ln (\epsilon)],
$$

where $u$ is the imaginary frequency, $V$ is the interelectron Coulomb interaction potential, and $\widetilde{\chi}$ is the local-field density response. The isotropic dielectric function, $\epsilon=\operatorname{tr}(1+4 \pi \alpha) / 3$, is also specified by the local-field density response, $\tilde{\chi}$ $=\nabla \cdot \alpha \cdot \nabla$. The nonlocal correlation energy is further approximated,

$$
E_{c}^{\mathrm{n} l}[n]=\frac{1}{2} \int d \mathbf{r} d \mathbf{r}^{\prime} n(\mathbf{r}) \phi\left(\mathbf{r}, \mathbf{r}^{\prime}\right) n\left(\mathbf{r}^{\prime}\right),
$$

through a kernel $\phi$ specified by a number of sum rules ${ }^{22}$ and physics results. $22,23,53$ The interaction energy [Eq. (3)] is consistently constructed to vanish for a homogeneous system. The kernel $\phi$ is specified by a pair of local parameters $q_{0}(\mathbf{r})$ and $q_{0}\left(\mathbf{r}^{\prime}\right)$ that depend on the electron density and the density gradient. The kernel can be tabulated in advance in terms of an effective separation, $D=\left[\left(q_{0}+q_{0}^{\prime}\right) / 2\right]\left|\mathbf{r}-\mathbf{r}^{\prime}\right|$, and an asymmetry parameter, $\delta=\left(q_{0}-q_{0}^{\prime}\right) /\left(q_{0}+q_{0}^{\prime}\right)$. The values of the $q_{0}$ 's are chosen to reproduce the (plasmon-pole) response of a weakly perturbed electron gas. ${ }^{22}$

With the evaluation of the nonlocal energy contribution (from underlying GGA calculations of the electron densities), we arrive at a vdW-DF total energy calculation, ${ }^{44}$

$$
\begin{gathered}
E^{\mathrm{vdW}-\mathrm{DF}}=E^{0}+E_{c}^{\mathrm{nl},} \\
E^{0}=E^{\mathrm{GGA}}-E_{c}^{\mathrm{GGA}}+E_{c}^{\mathrm{LDA}} .
\end{gathered}
$$

The GGA energy term $E^{\mathrm{GGA}}$ is here evaluated in the revPBE ${ }^{54}$ flavor (based on the self-consistent calculations for the electron density that we obtain in the PBE flavor of GGA). Effectively, this amounts to a small adjustment of the exchange contribution, which we do to minimize any potential artificial exchange binding in the plane-wave formalism used. ${ }^{20,22,44,55}$ The new (semilocal) energy contribution $E^{0}$ represents a modification of GGA that, for example, retains a description of the kinetic-energy repulsion as well as, for example, covalent ${ }^{34}$ or ionic $^{21}$ interactions.

\section{C. van der Waals density functional calculations}

The evaluation of the nonlocal correlation $E_{c}^{\mathrm{nl}}$ requires extra care due to a grid sensitivity of functional form (3). The $\mathrm{vdW}$ binding in the nanotube-crystal and dimer cases arises almost exclusively from a difference in nonlocal-correlation energy $E_{c}^{\mathrm{nl}}$ for the crystalline structure (dimer structure) and 
for the isolated nanotubes. However, the intramolecular density variation causes a very large contribution to $E_{c}^{\mathrm{nl}}$ that must be carefully subtracted in our $a b$ initio calculations of the internanotube interaction. Moreover, the evaluation of this intrananotube $E_{c}^{\mathrm{nl}}$ energy is somewhat sensitive to the relative position of atomic positions and FFT grid points. ${ }^{21}$ Nevertheless, robust and efficient evaluation of the internanotube binding is possible by simply ensuring that we calculate and subtract $E_{c}^{\text {nl }}$ contributions for the isolated nanotube using FFT grid points that closely match those of the composite system, as further explained in Ref. 21.

In practice, we evaluate the vdW-DF binding in the nanotube crystals and dimers by supplementing every interacting nanotube system by a suitable reference calculation of the isolated nanotube. We determine the binding in nanotube bundles by adding (at every nanotube separation) a DFT calculation of the electron density for a corresponding isolated nanotube located in a cell of double size in each of the two perpendicular directions and on a FFT grid that retains the absolute grid-point spacing. ${ }^{21}$ In our calculations of $E_{c}^{\mathrm{nl}}$, for the underlying GGA calculation, we use a FFT grid spacing that is always smaller than $0.14 \AA$ in any direction; this choice is found sufficient given our work to carefully synchronize the FFT gridding when we calculate the electron density for the interacting and isolated-nanotube system.

The real-space implementation of $E_{c}^{\mathrm{nl}}$ is simply applied to extended systems as graphite ${ }^{21}$ and polyethylene ${ }^{24}$ by evaluating $E_{c}^{\mathrm{nl}}$ for the unit-cell electron density, as well as the nonlocal interaction from its surrounding images. To accelerate the vdW-DF characterization, we limit the evaluation of the multidimensional integral [Eq. (3)] to contributions from grid points having a density larger than $10^{-4}$ a.u. The use of such a density cutoff is strongly motivated by the excellent convergence that we have previously documented for graphitic systems even when a significant ionic bond supplements the binding from nonlocal correlations. ${ }^{21}$ The nonlocal correction from the surrounding electron density rapidly converges in terms of the separation to the unit cell. We have tested that it is, in general, sufficient to only include the interaction from the electron density that is less than $12 \AA$ away from the unit-cell boundaries in the direction along the nanotube and $15 \AA$ in the directions perpendicular to the nanotube axis. Nevertheless, to converge the $E_{c}^{\mathrm{nl}}$ calculations to a sub-meV level and retain a very high relative accuracy even in the asymptotic regime, we choose to retain $E_{c}^{\mathrm{nl}}$ contributions originating from points closer than $24 \AA$ (and in some cases even closer than $30 \AA$ ) in the direction of the CNT extension.

\section{VAN DER WAALS INTERACTIONS AT INTERMEDIATE-TO- ASYMPTOTIC DISTANCES}

From our $a b$ initio vdW-DF calculations of the asymptotic van der Waals interactions, we extract an analytical determination of the van der Waals interaction energy $E_{\mathrm{vdW}}$ per unit length $L$ for a nanotube pair as a function of the separation $d=2 R_{\mathrm{geo}}+\Delta$. The analytical result for $E_{\mathrm{vdW}}(d) / L$ rests on the approximation summarized in Refs. 40,41 , and 50. It assumes that the electron densities of the two nanotubes do not overlap and constitute a lowest-order expansion ${ }^{56}$ of Eq. (2) in terms of the external-field susceptibilities, $\alpha_{\mathrm{eff}}$,

$$
E_{\mathrm{vdW}}=-\int_{0}^{\infty} \frac{d u}{2 \pi} i \operatorname{tr}\left[\alpha_{\mathrm{eff}, 1} T_{12} \alpha_{\mathrm{eff}, 2} T_{21}\right] .
$$

Here, $T_{i j}$ denotes the dipole interaction tensor, $T_{i j}=-\nabla_{i} \nabla_{j} \mid \mathbf{r}_{i}$ $-\left.\mathbf{r}_{j}\right|^{-1}$. The analysis is possible to carry out for nonisotropic external-field susceptibilities, ${ }^{40,41}$ but for an interpretation of our vdW-DF calculations of CNT interactions, it is sufficient to consider isotropic susceptibilities $\alpha_{\text {eff }}$. We focus on the interaction regime where effects of the CNT morphology dominate the variation in $E_{c}^{\mathrm{nl}} \approx E_{\mathrm{vdW}}$ with distance. ${ }^{40,50} \mathrm{We}$ assume a long-wavelength form of $\alpha_{\text {eff }}$ so that the resulting analytical determination remains valid at such intermediateto-asymptotic interaction distances.

The physics of the local-field and external-field susceptibilities defines the parametrization of our vdW-DF method. ${ }^{20,22,23,43}$ The long-wavelength electrodynamical response determines the interaction at large distances ${ }^{44}$ and our vdW-DF method describes this response by the isotropic effective (external-field) susceptibility, ${ }^{22}$

$$
\alpha_{\mathrm{eff}}^{g g}(u ; \mathbf{r})=\frac{n(\mathbf{r})}{u^{2}+\left[9 q_{0}^{2}(\mathbf{r}) /(8 \pi)\right]^{2}} .
$$

We stress that our extraction of this long-wavelength form serves only to establish formal connection between the full vdW-DF calculations and the analytical approximation. We also emphasize that neither the effective response [Eq. (7)] nor the full vdW-DF response function ${ }^{22}$ is explicitly designed to accurately reproduce, for example, the static dielectric response, which is in contrast to the functional approaches described in Refs. 20, 40, 41, and 44. Rather, the full vdW-DF response function ${ }^{22}$ is exclusively constructed from an ansatz for the plasmon-pole response, conservation rules, and many-body calculations ${ }^{22,53}$ to describe the average response. The full vdW-DF description involves contributions from different frequencies and wavelengths and it is the average response, rather than the long-wavelength limit [Eq. (7)], that determines the interactions at binding separations where our vdW-DF approach is most needed

Our analysis focus on the intermediate-to-asymptotic separations furthermore allows us to consider the contributions to the susceptibility from the electron density averaged over the angular and along-tube variations. We thus substitute $\alpha_{\text {eff }}(\mathbf{r} ; u) \rightarrow \bar{\alpha}_{\text {eff }}(s ; u)$, where $s$ denotes the radial distance from the nanotube center. The nanotube interaction per unit length, given in terms of the effective response [Eq. (7)], becomes $^{41}$

$$
\begin{aligned}
\frac{E_{\mathrm{vdW}}}{L}=- & \int_{0}^{\infty} \frac{d u}{2 \pi} \int_{0}^{\infty} d s_{1} s_{1} \int_{0}^{\infty} d s_{2} s_{2} \bar{\alpha}_{\mathrm{eff}}\left(s_{1} ; u\right) \bar{\alpha}_{\mathrm{eff}}\left(s_{2} ; u\right) \\
& \times \sum_{\alpha, \beta=s, \theta, z} G_{\alpha, \beta}\left(s_{1}, s_{2}\right),
\end{aligned}
$$

with the geometry factors, 


$$
\begin{aligned}
G_{\alpha \beta}= & \int_{0}^{2 \pi} d \theta_{1} \int_{0}^{2 \pi} d \theta_{2} \int_{0}^{\infty} d\left(z_{2}-z_{1}\right) \\
& \times\left[T_{12}^{\alpha \beta}\left(s_{1}, \theta_{1}, z_{1} ; s_{2}, \theta_{2}, z_{2}\right)\right]^{2},
\end{aligned}
$$

where the dipole interaction tensors are expressed in cylindrical coordinates. The result [Eq. (8)] is easily expanded in the inverse center-of-mass separation $d^{-1}$ of the nanotubes, yielding interaction energies of the following form:

$$
\frac{E_{\mathrm{vdW}}}{L}=-\frac{B_{5}}{d^{5}}-\frac{B_{7}}{d^{7}}+\cdots,
$$

where $B_{5}$ and $B_{7}$ are given by

$$
\begin{gathered}
B_{5}=\frac{9}{8} \int_{0}^{\infty} d u \Xi^{(0)}(u)^{2}, \\
B_{7}=\frac{225}{16} \int_{0}^{\infty} d u \Xi^{(0)}(u) \Xi^{(2)}(u) .
\end{gathered}
$$

Here,

$$
\Xi^{(i)}(u) \equiv \int_{0}^{\infty} d s 2 \pi s \bar{\alpha}_{\mathrm{eff}}^{g g}(s ; u) s^{i}
$$

is simply the $i$ th moment of the effective response. We calculate the coefficients $B_{5,7}$ directly from the $\alpha_{\text {eff }}(s ; u)$ variation specified by our underlying GGA-DFT calculations of the CNT electron-density variation. We have explicitly tested consistency of this asymptotic evaluation and the set of full vdW-DF calculations for $\Delta>16-20 \AA$.

The relevant external-field electrodynamical response $\left(\alpha_{\text {eff }}\right)$ of the nanotubes is dominated by contributions at some radius $R>R_{\text {geo }}$. This is the experience gained from describing the electrodynamical response and van der Waals interactions of surfaces ${ }^{57}$ and from previous investigation of the van der Waals bonding in graphitics. ${ }^{20,21}$ While the results presented in Refs. 40, 41, and 50 made the assumption that the response $\alpha_{\text {eff }}$ exclusively arose from the atom wall (at $R_{\text {geo }}$ ), the formal special-function evaluation, ${ }^{41,58}$

$$
\frac{E_{\mathrm{vdW}}(d, R)}{L}=-\frac{B_{5}}{d^{5}{ }^{3}} F_{2}\left(\frac{1}{2}, \frac{5}{2}, \frac{5}{2} ; 1,1 ; \frac{4 R^{2}}{d^{2}}\right),
$$

is possible as long as we may assume the response $\alpha_{\text {eff }}$ dominated by contributions at any (single) radius $R$. The interaction result [Eq. (14)] simply reflects the morphology (interaction of two hollow cylinders). The effective vdW-DF response $\alpha_{\text {eff }}^{g g}(s ; u)$ is dominated by contributions at $R_{\text {geo }}$ and outside in the CNT density tails. In this paper, we choose a value for the effective response radius,

$$
R_{\text {eff }}=\sqrt{\frac{2 B_{7}}{25 B_{5}}}
$$

and obtain an analytical approximation,

$$
E_{c}^{\mathrm{nl}}(\Delta) \approx E_{\mathrm{vdW}}\left(d=2 R_{\mathrm{geo}}+\Delta, R_{\mathrm{eff}}\right),
$$

that exactly reproduces the asymptotic variation of the full vdW-DF calculations up to the second spatial moment given by $B_{5}$ and $B_{7}$. The relative position of $R_{\text {eff }}$ and $R_{\text {geo }}$ are shown in Fig. 2.

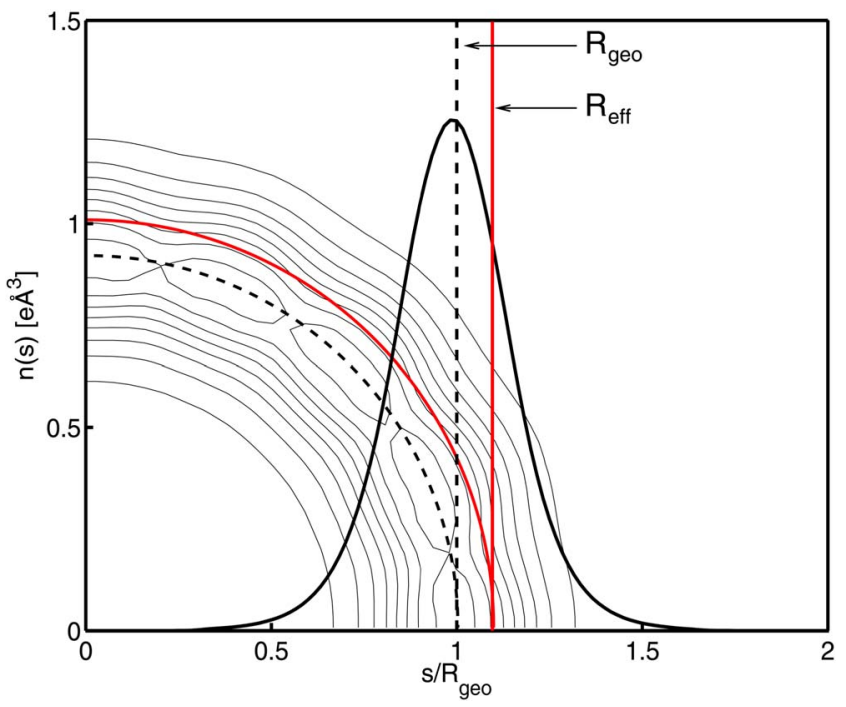

FIG. 2. (Color online) Length and radially averaged electron density $\bar{n}(s)$ shown together with the positions of the effective and the geometric radius. The radial separation is given relative to the geometric radius of the nanotube. The background inset shows the length-averaged electron density with contour lines separated in steps of $0.15 e \AA^{3}$.

\section{RESULTS AND DISCUSSION}

\section{A. van der Waals bonding in a nanotube crystal}

The top panel of Fig. 3 reports our ab initio calculation (thick solid curve) of the vdW-DF binding in a hexagonal crystal of semiconducting $(8,0)$ nanotubes. The vdW-DF result for the binding separation, $\Delta_{\text {bind }} \approx 3.45 \AA$, is in very good agreement with experimental observations, ${ }^{4-7} \Delta_{\text {expt }}$ $\approx 3.4 \AA$, and corrects the poor structure predicted in traditional LDA calculations, ${ }^{28} \Delta_{\text {bind }}^{\mathrm{LDA}}=3.1 \AA$. The binding energy for the nanotube bundle is large, $E_{\text {bind }}^{\text {crys }}=-30 \mathrm{meV} /$ atom, corresponding to $-0.225 \mathrm{eV} / \AA$, which is consistent with interaction strengths that we have previously calculated in $\mathrm{vdW}-\mathrm{DF}$ for the interlayer binding in graphite, ${ }^{21}$ $-50 \mathrm{meV} /$ atom. The vdW-DF binding energy is significantly larger than the LDA result, ${ }^{28} \approx 10 \mathrm{meV} /$ atom, obtained for a metallic $(6,6)$ nanotube.

The figure documents differences between the vdW-DF calculation for the full CNT crystal (thick solid curve) and corresponding approximations based on CNT-pair contributions (thin solid curve). The regular vdW-DF calculations yield a CNT-crystal binding energy that is larger than the binding-energy estimate obtained from the summation of pair contributions, $E_{\text {bind }}^{\text {crysest }}=-29 \mathrm{meV} /$ atom, corresponding to $-0.220 \mathrm{eV} / \AA$. We find that the vdW-DF energy difference $E_{\text {bind }}^{\text {crys }}-E_{\text {bind }}^{\text {crys,est }}$ is split evenly between contributions $E_{c}^{\text {nl }}$ and $E_{0}$.

Nevertheless, the vdW-DF results for the pair-interaction energies constitute a fair approximation of the hexagonal ordering arising in the nanotube bundles. It is thus possible to use vdW-DF calculations of the CNT-pair interactions at general (parallel) configurations (of different relative rotations) to model the cohesion and binding in more general nanotube structures such as yarn and rope. 


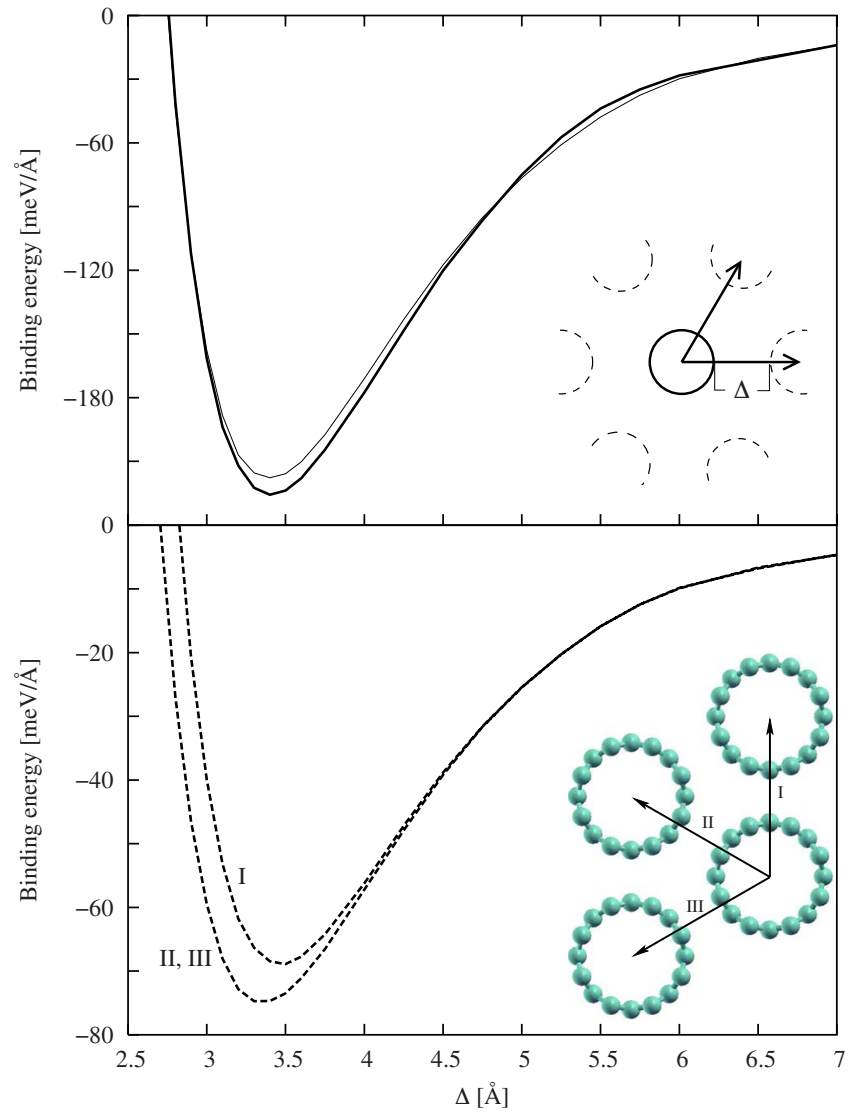

FIG. 3. (Color online) Nanotube binding energy $E^{\mathrm{vdW}-\mathrm{DF}}(\Delta)$ $-E^{\mathrm{vdW}-\mathrm{DF}}(\Delta \rightarrow \infty)$ (per unit length) for semiconducting $(8,0)$ nanotubes evaluated in vdW-DF as a function of the wall-to-wall separation $\Delta$. The top panel shows vdW-DF results for the hexagonal crystal and compares the crystal interaction energy (thick solid curve) against an estimate (thin solid curve) based on a sum of vdW-DF results for the nanotube-pair interactions. The bottom panel reports the vdW-DF calculations of the binding of two parallel nanotubes (dashed curves) in three different atomic configurations indicated in the inset. The sum of those three pair interactions constitutes the approximation for the crystal interaction (thin solid curve) in the top panel.

\section{B. van der Waals bonding in a pair of parallel semiconducting nanotubes}

Figure 3, bottom panel, reports our ab initio calculation of the vdW bonding between pairs of parallel $(8,0)$ nanotubes at three configurations "I," "II," and "III" identified in the inset. These are the configurations that are relevant for the pairinteraction estimate of the CNT hexagonal crystal (thin solid curve in top panel). Even for a CNT pair, the nanotube binding is very significant, $E_{\text {bind }}^{\text {pair }} \approx-9.2 \mathrm{meV} /$ atom, but occurs at slightly different binding separations for different relative nanotube rotations. We find that the vdW-DF results for the nonlocal correlation term $E_{c}^{\mathrm{nl}}$ are almost identical (smaller than $1 \%$ variation outside binding separations) for the three CNT-pair configurations. As is evident in the inset (which identifies actual atomic organization investigated in our vdW-DF method), the atomic organization is in better registry for some organization than others. There consequently exists some electron-density variation with the rotations, and our vdW-DF method is sensitive to this variation since the semilocal contribution $E_{0}$ contains a description of the kinetic-energy repulsion.

As an interesting aside, we note that the high symmetry of the semiconducting $(8,0)$ nanotube permits us to test the grid sensitivity and consistency of the vdW-DF calculations. There must exist a fourfold symmetry in atomic positions around the $(8,0)$ nanotube and such an approximate symmetry also emerges as a result of the initial atomic relaxation that we perform for an individual nanotube in traditional DFT. The symmetry implies a periodicity $\pi$ in the variation of the vdW binding between a pair of nanotubes with the relative rotation angle $\Theta$. However, the imperfect relaxation causes small variations in the exact atomic location relative to the grid. We find that the vdW-DF calculations are more sensitive than the underlying traditional-DFT calculations. Nevertheless, the vdW-DF calculations respect the symmetry and produce $\mathrm{vdW}$ interaction energies for $\Theta$ and $\Theta+\pi$ relative rotations that are identical even at a sub-meV energy scale.

\section{Approximative microscopic modeling for general nanotube- bundle structures}

The comparison between the vdW-DF results for the nanotube crystal and for the approximation based on a sum of nanotube-pair interactions (Fig. 3, top panel) suggests a framework for an approximative microscopic modeling for the binding in more general bundles of (semiconducting) nanotubes. A simple mapping of the binding energy for two parallel nanotubes for all combinations of independent rotations (relative to the interaction line) provides the starting point. Adding such general pair-interaction contributions allows vdW-DF calculations to account for general vdW bonding in aligned nanotube structures, including nanotube yarn and ropes.

Moreover, the finding of insignificant differences between the $E_{c}^{\mathrm{nl}}$ energy contributions for the three nanotube-pair configurations investigated in Fig. 3 suggests an additional speed up in the modeling. Assuming that general, independent nanotube rotations also cause insignificant $E_{c}^{\mathrm{nl}}$ differences, it is sufficient to supplement one calculation of $E_{c}^{\mathrm{nl}}$ (detailed below) with a mapping of the general $E^{0}$ variation. This can be obtained at a computational cost equal to that of traditional implementations of DFT. A forthcoming study will provide vdW-DF results for the bundling of a broader set of semiconducting nanotubes and an explicit test of the $E^{\mathrm{vdW}-\mathrm{DF}}$ and $E_{c}^{\mathrm{nl}}$ variation with general (independent) nanotuberotation angles to detail the suggested approximative modeling approach.

\section{Nature of the van der Waals bonding at bundle and at intermediate separations}

Figure 4 compares the full vdW-DF calculation of the $E_{c}^{\mathrm{nl}}$ contribution to the CNT-pair interaction (thick dashed curves) with vdW-DF based approximations $E_{\mathrm{vdW}}$ (dotted and dash-dotted curves) near binding separations (main panel) and in the intermediate-to-asymptotic regime (inset). The contribution $E_{c}^{\mathrm{nl}}$ is evaluated for the configuration I 


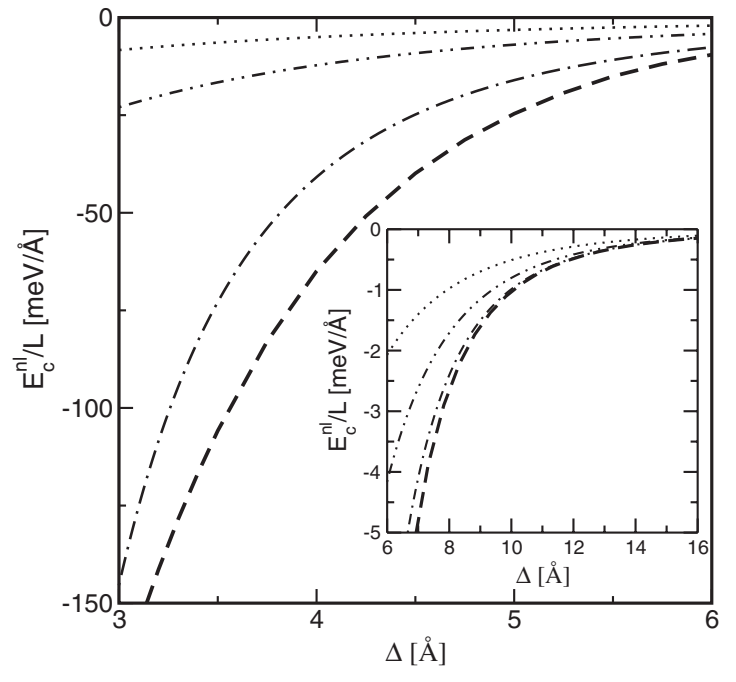

FIG. 4. Nonlocal correlation energy per unit length for a nanotube dimer near binding separations (main panel) and in the intermediate-to-asymptotic regime (inset panel). The thick dashed curves show the results of the full vdW-DF calculation of the nonlocal correlation energy (vdW interaction). The dotted and dasheddouble-dotted curves show the (traditional) asymptotic interaction estimates as determined from the asymptotics $-B_{5} d^{-5}$ and $-B_{5} d^{-5}$ $-B_{7} d^{-7}$, respectively. Finally, the dashed-dotted curve shows the analytical evaluation that approximates the electrodynamical response by the long-wavelength form but retains a full description of the nanotube morphology. The analytical result also reflects the surface-physics insight that the electrodynamical response is dominated by contributions outside the radius defined by the atomic positions (Fig. 2).

shown in the inset of the lower panel of Fig. 3 (but $E_{c}^{\text {nl }}$ exhibits only insignificant differences between configurations I, II, and III). All of the estimates $E_{\mathrm{vdW}}$ are, of course, independent of the nanotube rotation by construction. The dashed-dotted curves show the analytical CNT-pairinteraction estimate [Eq. (16)] that invokes a longwavelength form of the electrodynamical response but respects the morphology of the interaction problem. ${ }^{40,41}$ The dotted and dashed-double-dotted curves show (for $d=\Delta$ $\left.+2 R_{\text {geo }}\right)$ traditional interaction estimates $-B_{5} / d^{5}$ and $-B_{5} / d^{5}-B_{7} / d^{7}$, respectively. The traditional interaction estimates clearly only become applicable in a very remote asymptotic regime beginning at $\Delta>16 \AA$.

The main panel shows that the full vdW-DF calculations are necessary around the binding separations $\Delta_{\text {bind }} \sim 3.5 \AA$. Here, the interaction is significantly enhanced compared with estimates based on the asymptotic dipolar response. The enhancement is consistent with the behavior documented for graphite interactions, as described in an earlier generation of vdW-DF. ${ }^{43}$ The enhancement relative to the analytical approximations persists even beyond separations $(\Delta \approx 4 \AA)$ when an overlap of electron densities no longer exists. It arises in part because the complete interaction also contains multipole interactions. ${ }^{20}$

However, the contrast between the main panel and the inset panel in Fig. 4 also documents a qualitative change in nature in the mutual interaction with increasing separation. Gradually, there is a transition in an intermediate-toasymptotic regime (shown inset panel) where the mutual interaction is essentially specified by the morphology of the nanotube density variation, as summarized in the analytical interaction estimate [Eq. (16)].

\section{CONCLUSIONS}

We have presented $a b$ initio calculations of the binding in nanotube bundles and in nanotube dimers. Our calculations rest on a density functional description ${ }^{22}$ that includes accounts of the dispersive forces. We have, in addition, presented an analytical evaluation valid at intermediate to large nanotube separation.

Our microscopic theory of the CNT binding of semiconducting $(8,0)$ CNTs provides a number of results based on the $a b$ initio vdW-DF calculations. The CNT study supplements recent microscopic theory studies of elements of the DNA base-pair interaction ${ }^{26}$ and of the polyethylene polymer crystal $^{24}$ in a broader goal of developing a microscopic theory of self-organization and bundling of nanoscale filaments. This vdW-DF study finds a nanotube wall-to-wall separation in very good agreement with experiments and predicts a vdW bonding with a significant strength, which is consistent with recent measurements for graphitics. ${ }^{29}$

Our work furthermore constitutes an analysis that details the nature of the mutual CNT interactions by identifying a set of distinct interaction regimes. We provide an analytical approximation for the CNT-pair interactions at distances when the electron densities are nonoverlapping and the dielectric responses are dominated by the long-wavelength form. Comparing against our $a b$ initio vdW-DF calculations (valid at general distances), we thereby identify a relatively broad intermediate-to-asymptotic regime where the interaction form is primarily defined by the CNT morphology.

Finally, this introductory study also suggests a framework for an efficient implementation of quantum-physical modeling of the CNT bundling in more general geometries, including nanotube yarn and ropes. The vdW-DF study documents that a summation of nanotube-pair-interaction energies represents a fair approximation for the nanotube-crystal binding energy when the CNT-pair interaction is calculated in vdWDF. A simple vdW-DF mapping of the nanotube-pair interaction for general (independent) CNT rotations relative to the interaction axis therefore provides adequate input for describing the vdW bonding in general aligned CNT structures.

\section{ACKNOWLEDGMENTS}

We thank B. I. Lundqvist for useful discussions and L. Glasser for suggesting and detailing the special-function evaluation [Eq. (14)] from our corresponding parallelnanotube interaction result in Ref. 50. Support from the Swedish Research Council, the Swedish Foundation for Strategic Research, the Swedish National Graduate School in Materials Science, as well as allocation of computer time at SNIC (Swedish National Infrastructure for Computing) is gratefully acknowledged. 
*hyldgaar@chalmers.se

${ }^{1}$ M. S. Dresselhaus, G. Dresselhaus, and Ph. Avouris, Carbon Nanotubes, Synthesis, Structure, Properties, and Applications (Springer-Verlag, Berlin, 2001).

${ }^{2}$ M. M. Treacy, T. Ebbesen, and J. M. Gibson, Nature (London) 381, 678 (1996); J. P. Lu, Phys. Rev. Lett. 79, 1297 (1997).

${ }^{3}$ R. R. Schlittler, J. W. Seo, J. K. Gimzewski, C. Durkan, M. S. Saifullah, and M. E. Welland, Science 292, 1136 (2001).

${ }^{4}$ S. Iijima, Nature (London) 354, 56 (1991).

${ }^{5}$ X. F. Zhang, X. B. Zhang, G. Van Tendeloo, S. Amelinckx, M. Op de Beeck, and J. Van Landuyt, J. Cryst. Growth 130, 368 (1993).

${ }^{6}$ M. Terrones, N. Grobert, J. Olivares, J. P. Zhang, H. Terrones, K. Kordatos, W. K. Hsu, J. P. Hare, P. D. Townsend, K. Prassides, A. K. Cheetham, H. W. Kroto, and D. R. M. Walton, Nature (London) 388, 52 (1997).

${ }^{7}$ C.-H. Kiang, M. Endo, P. M. Ajayan, G. Dresselhaus, and M. S. Dresselhaus, Phys. Rev. Lett. 81, 1869 (1998).

${ }^{8}$ Y.-L. Li, I. Kinloch, and A. H. Windle, Science 304, 276 (2004).

${ }^{9}$ M. Zhang, K. R. Atkinson, and R. H. Baughman, Science 306, 1358 (2004).

${ }^{10}$ L. M. Ericson et al., Science 305, 1447 (2004).

${ }^{11}$ J. Hwang, H. H. Gommans, A. Ugawa, H. Tashiro, R. Haggenmueller, K. I. Winey, J. E. Fischer, D. B. Tanner, and A. G. Rinzler, Phys. Rev. B 62, R13310 (2000).

${ }^{12}$ X. Blase, L. X. Benedict, E. L. Shirley, and S. G. Louie, Phys. Rev. Lett. 72, 1878 (1994); D. Sánchez-Portal, E. Artacho, J. M. Soler, A. Rubio, and P. Ordejón, Phys. Rev. B 59, 12678 (1999).

${ }^{13}$ See, for example, V. Barone and G. E. Scuseria, J. Chem. Phys. 121, 10376 (2004).

${ }^{14}$ For example, in the parametrization, J. P. Perdew and A. Zunger, Phys. Rev. B 23, 5048 (1981).

${ }^{15}$ J. P. Perdew, K. Burke, and M. Ernzerhof, Phys. Rev. Lett. 77, 3865 (1996).

${ }^{16}$ A. Kis, G. Csányi, J.-P. Salvetat, T.-N. Lee, E. Couteau, A. J. Kulik, W. Benoit, J. Brugger, and L. Ferró, Nat. Mater. 3, 153 (2004).

${ }^{17}$ For a description of corresponding LDA problems in providing a transferable description for polymer crystals, please see M. S. Miao et al., J. Chem. Phys. 115, 11317 (2001).

${ }^{18}$ J. M. Pérez-Jordá and A. D. Becke, Chem. Phys. Lett. 233, 134 (1995).

${ }^{19}$ H. Rydberg, N. Jacobson, P. Hyldgaard, S. I. Simak, B. I. Lundqvist, and D. C. Langreth, Surf. Sci. 532-535, 606 (2003).

${ }^{20}$ H. Rydberg, M. Dion, N. Jacobson, E. Schröder, P. Hyldgaard, S. I. Simak, D. C. Langreth, and B. I. Lundqvist, Phys. Rev. Lett. 91, 126402 (2003).

${ }^{21}$ E. Ziambaras, J. Kleis, E. Schröder, and P. Hyldgaard, Phys. Rev. B 76, 155425 (2007).

${ }^{22}$ M. Dion, H. Rydberg, E. Schröder, D.C. Langreth, and B.I. Lundqvist, Phys. Rev. Lett. 92, 246401 (2004); 95 109902(E) (2005).

${ }^{23}$ T. Thonhauser, V. R. Cooper, S. Li, A. Puzder, P. Hyldgaard, and D. C. Langreth, Phys. Rev. B 76, 125112 (2007).

${ }^{24}$ J. Kleis, B. I. Lundqvist, D. C. Langreth, and E. Schröder, Phys. Rev. B 76, 100201(R) (2007).

${ }^{25}$ See, for example, G. Avitabile et al., J. Polym. Sci., Polym. Lett. Ed. 13, 351 (1975).

${ }^{26}$ V. R. Cooper, T. Thonhauser, A. Puzder, E. Schröder, B. I. Lundqvist, and D. C. Langreth, J. Am. Chem. Soc. 130, 1304 (2008);
A. Puzder, M. Dion, and D. C. Langreth, J. Chem. Phys. 124, 164105 (2006); T. Thonhauser, A. Puzder, and D. C. Langreth, ibid. 124, 164106 (2006).

${ }^{27}$ A quick set of DFT-GGA calculations produce an extremely shallow binding indication at much too large distances and representing only $5 \%$ of the presently report vdW-DF binding.

${ }^{28}$ J.-C. Charlier, X. Gonze, and J.-P. Michenaud, Europhys. Lett. 29, 43 (1995); S. Reich, C. Thomsen, and P. Ordejón, Phys. Rev. B 65, 155411 (2002).

${ }^{29}$ R. Zacharia, H. Ulbricht, and T. Hertel, Phys. Rev. B 69, 155406 (2004).

${ }^{30}$ J. Q. Sun and R. J. Bartlett, J. Chem. Phys. 104, 8553 (1996); S. Hirata and S. Iwata, ibid. 109, 4147 (1998); S. Suhai, Phys. Rev. B 27, 3506 (1983).

${ }^{31}$ P. Y. Ayala and G. E. Scuseria, J. Chem. Phys. 110, 3660 (1999).

${ }^{32}$ P. Y. Ayala, K. N. Kudin, and G. E. Scuseria, J. Chem. Phys. 115, 9698 (2001).

${ }^{33}$ S. D. Chakarova-Käck, E. Schröder, B. I. Lundqvist, and D. C. Langreth, Phys. Rev. Lett. 96, 146107 (2006).

${ }^{34}$ S. D. Chakarova-Käck, Ø. Borck, E. Schröder, and B. I. Lundqvist, Phys. Rev. B 74, 155402 (2006).

${ }^{35}$ A parallel project investigates the adhesion of graphite on $\mathrm{SiC}$ in a surface unit cell that has the graphite-sheet overlayer relaxed to $2 \%$ strain; E. Ziambaras, C. Ruberto, B. I. Lundqvist, and P. Hyldgaard (unpublished).

${ }^{36}$ Y. U. Barash, and O. I. Notysh, Zh. Eksp. Teor. Fiz. 98, 542 (1990).

${ }^{37}$ B. E. Sernelius and P. Björk, Phys. Rev. B 57, 6592 (1998).

${ }^{38}$ M. Boström and B. E. Sernelius, Phys. Rev. B 61, 2204 (2000).

${ }^{39}$ J. F. Dobson, A. White, and A. Rubio, Phys. Rev. Lett. 96, 073201 (2006).

${ }^{40}$ E. Schröder and P. Hyldgaard, Surf. Sci. 532, 880 (2003).

${ }^{41}$ J. Kleis, P. Hyldgaard, and E. Schröder, Comput. Mater. Sci. 33, 192 (2005).

${ }^{42}$ DACAPO from http://wiki.fysik.dtu.dk/dacapo/

${ }^{43}$ H. Rydberg, B. I. Lundqvist, D. C. Langreth, and M. Dion, Phys. Rev. B 62, 6997 (2000).

${ }^{44}$ D. C. Langreth, M. Dion, H. Rydberg, E. Schröder, P. Hyldgaard, and B. I. Lundqvist, Int. J. Quantum Chem. 101, 599 (2005).

${ }^{45}$ W. Kohn, Y. Meir, and D. E. Makarov, Phys. Rev. Lett. 80, 4153 (1998).

${ }^{46}$ S. Kurth and J. P. Perdew, Phys. Rev. B 59, 10461 (1999).

${ }^{47}$ J. F. Dobson and J. Wang, Phys. Rev. Lett. 82, 2123 (1999); J. F. Dobson and J. Wang, Phys. Rev. B 62, 10038 (2000); J. F. Dobson and B. P. Dinte, Phys. Rev. Lett. 76, 1780 (1996).

${ }^{48}$ J. M. Pitarke and J. P. Perdew, Phys. Rev. B 67, 045101 (2003).

${ }^{49}$ For illustrations of applications to model, molecular, and/or solid systems, see, for example, J. Jung, P. Garcia-Gonzalez, J. F. Dobson, and R. W. Godby, Phys. Rev. B 70, 205107 (2004); F. Furche and T. Voorhis, J. Chem. Phys. 122, 164106 (2005); M. Fuchs and X. Gonze, Phys. Rev. B 65, 235109 (2002); M. Fuchs, K. Burke, Y.-M. Niquet, and X. Gonze, Phys. Rev. Lett. 90, 189701 (2003); F. Aryasetiawan, T. Miyake, and K. Terakura, ibid. 88, 166401 (2002); T. Miyake, F. Aryasetiawan, T. Kotani, M. van Schilfgaarde, M. Usuda, and K. Terakura, Phys. Rev. B 66, 245103 (2002); A. Marini, P. Garcia-Gonzalez, and A. Rubio, Phys. Rev. Lett. 96, 136404 (2006).

${ }^{50}$ E. Schröder and P. Hyldgaard, Mater. Sci. Eng., C 23, 721 (2003).

${ }^{51}$ C. Amovilli and N. H. March, Carbon 43, 1634 (2005). 
${ }^{52}$ H. J. Monkhorst and J. D. Pack, Phys. Rev. B 13, 5188 (1976).

${ }^{53}$ D. C. Langreth and S. H. Vosko, Adv. Quantum Chem. 21, 175 (1990).

${ }^{54}$ Y. Zhang and W. Yang, Phys. Rev. Lett. 80, 890 (1998).

${ }^{55}$ X. Wu, M. C. Vargas, S. Nayak, V. Lotrich, and G. Scoles, J. Chem. Phys. 115, 8748 (2001).

${ }^{56}$ B. I. Lundqvist, Y. Andersson, H. Shao, S. Chan, and D. C. Langreth, Int. J. Quantum Chem. 56, 247 (1995).
${ }^{57}$ See, for example, E. Zaremba and W. Kohn, Phys. Rev. B 13, 2270 (1976); B. N. J. Persson and P. Apell, ibid. 27, 6058 (1983); B. N. J. Persson and E. Zaremba, ibid. 30, 5669 (1984); A. Liebsch, ibid. 33, 7249 (1986); E. Hult, H. Rydberg, B. I. Lundqvist, and D. C. Langreth, ibid. 59, 4708 (1999); E. Hult, P. Hyldgaard, J. Rossmeisl, and B. I. Lundqvist, ibid. 64, 195414 (2001).

${ }^{58}$ L. Glasser (private communication). 